\title{
Relação entre mês de nascimento e estatura de atletas do mundial de futebol sub 17
}

\section{Relationship between month of birth and height of athletes in the under 17 World Cup}

\author{
Eduardo Macedo Penna ${ }^{1}$ \\ Renato Melo Ferreira ${ }^{1}$ \\ Varley Teoldo da Costa ${ }^{1}$ \\ Bruna Silva Santos ${ }^{1}$ \\ Luiz Carlos Couto de Albuquerque Moraes ${ }^{1}$
}

Resumo - O Efeito da Idade Relativa que consiste na vantagem obtida pelo atleta nascido mais próximo ao início do ano de seleção, mostra-se uma variável importante para o desenvolvimento de talentos, particularmente, devido aos aspectos maturacionais. Objetivou-se analisar a distribuição das datas de nascimento dos atletas de futebol, participantes do mundial sub17, e verificar diferenças na estatura dos mesmos. Para isso, foram coletados junto ao site oficial da FIFA os dados referentes aos 1479 atletas inscritos nas últimas três competições $(2007,2009$ e 2011). Posteriormente, os dados foram divididos pelo quartil da data de nascimento e também, pela zona de posição do campo. Para a comparação da representatividade proporcional de cada quartil da data de nascimento, foi utilizado o teste de qui-quadrado com correção de Bonferroni. Para a comparação das estaturas por quartil da data de nascimento e setor do campo, utilizou-se a análise de variância ANOVA em esquema fatorial 2x2. Também foi empregada uma análise de correlação de Spearman para a verificação de associação entre as variáveis. Diferenças foram encontradas na distribuição dos quartis da data de nascimento, com predomínio de atletas nascidos no primeiro e segundo quartis da data de nascimento, e nas estaturas dos atletas, sendo que os atletas nascidos no primeiro e segundo quartis da data de nascimento apresentaram estatura média superior. Concluiu-se que o efeito da idade relativa é um fator que pode influenciar a seleção de jogadores de futebol e que influencia a estatura (variável maturacional).

Palavras-chave: Atletas; Futebol; Grupos etários; Talento.

Abstract - The Relative Age Effect, which consists in the advantage obtained by an athlete born closer to the beginning of the year selection, is shown as an important variable for the development of talent, particularly due to maturational aspects. The objective of this study was to analyze the distribution of the birth dates of the soccer players participating in the Under 17 World Championship, and verify the differences in their height. In order to do so, data referring to 1479 athletes registered in the last three competitions (2007, 2009 and 2011) was collected from the official FIFA site. Subsequently, the corresponding data was divided into birth date quartiles and also zone position of the field. For the comparison of proportional representativeness of each birth date quartile, it was used the chi-squared test with Bonferroni correction. The ANOVA factorial $2 \times 2$ was applied to compare the heights by birth date quartile and by zone position of the field. A Spearman correlation analysis was also used to verify the association between the variables. Differences were found in the distribution of birth date quartiles, with a predominance of athletes born in the first and second quartiles; and in the height of the athletes, having athletes who were born in the first and second quartiles a higher average height. It was concluded that the relative age effect is a factor that may influence the selection of soccer players and that influences the height ( $a$ maturational variable).

Key words: Age Groups; Athletes; Soccer; Talent.
1 Universidade Federal de Minas Gerais. Escola de Educação Física, Fisioterapia e Terapia Ocupacional. Laboratório de Psicologia do Esporte. Belo Horizonte, MG. Brasil.

Recebido em 27/07/11 Revisado em 06/01/12 Aprovado em 21/06/12 


\section{INTRODUÇÃO}

Dentre os vários fatores que podem influenciar o desempenho esportivo, pode-se citar a diferença na idade cronológica entre atletas de um mesmo grupo. Essa variável é importante devido às consequências do que os pesquisadores denominam Efeito da Idade Relativa, e atualmente, vem recebendo especial atenção da comunidade científica do esporte ${ }^{1-5}$.

Segundo dados de pesquisas em diferentes $\operatorname{contextos}^{6-9}$, o Efeito da Idade Relativa apresenta-se expressivamente no futebol. Isso pode ocorrer, segundo a literatura, devido à grande popularidade da modalidade e sua consequente competitividade e também, devido à sua organização baseada em categorias etárias que duram dois anos ${ }^{5,10}$. Essa estruturação faz com que a diferença etária entre crianças da mesma categoria possa alcançar, em um caso extremo, até 23 meses, gerando, assim, uma vantagem física e cognitiva para os atletas que nasceram no início do primeiro ano da categoria, o que aumenta as chances destas chegarem ao alto nível de desempenho ${ }^{11}$.

Ainda com relação ao Efeito da Idade Relativa, sabe-se que aqueles atletas favorecidos por essa variável são apontados como mais talentosos e significativamente mais selecionados pelos treinadores para as equipes de futebol $^{3,5,6}$. Observa-se, também, a influência do Efeito da Idade Relativa em outras modalidades ${ }^{12,13}$. Esses atletas favorecidos também são mais convocados para os jogos e participam por mais tempo das partidas do que seus pares nascidos posteriormente ${ }^{4}$. Além disso, em dez países diferentes da Europa, Helsen et al. ${ }^{7}$ verificaram uma maior representação de atletas, nascidos no início do ano, convocados para as seleções nacionais das categorias sub 15, sub 16 e sub 18 .

A vantagem na idade relativa, principalmente relacionada com a maturação física precoce, parece estar ligada ao sucesso dos atletas, especialmente nas modalidades em que existe uma preferência por indivíduos de maior tamanho corporal ${ }^{14}$, e no caso do futebol, principalmente para atletas que atuam em determinadas posições do campo ${ }^{15}$.

No estudo de Hansen et al. ${ }^{14}$ com jovens atletas de futebol, foram identificadas preferências dos clubes de elite por atletas com maior estatura e níveis maturacionais mais avançados. Os autores também identificaram que atletas de menor estatura eram selecionados por clubes considerados de menor expressão. Já o estudo de Gall et al..$^{15}$, que avaliou 161 jovens atletas participantes de um centro de formação de atletas de futebol, encontrou diferenças significativas na massa corporal em goleiros e na massa corporal e estatura em defensores que conseguiram alcançar o mais alto nível competitivo, em comparação com aqueles que permaneceram no amadorismo e não foram incorporados a equipes profissionais. Com base no exposto pela literatura, a variável Efeito da Idade Relativa no futebol relacionada com os aspectos maturacionais, em especial a estatura, tem sido avaliada analisando atletas pertencentes a clubes de formação esportiva no futebol. Entretanto, as análises destas variáveis com atletas pertencentes a seleções nacionais que disputam as principais competições internacionais promo- 
vidas pela FIFA, carecem de investigações por parte dos pesquisadores para que se possa avaliar e identificar se os efeitos relacionados à Idade Relativa neste nível de exigência competitiva são semelhantes ou não aos efeitos identificados nas competições clubísticas.

Dessa forma, este estudo apresenta como objetivo analisar a distribuição das datas de nascimento dos atletas de futebol participantes do mundial sub-17, nas edições 2007, 2009 e 2011, e verificar a diferença na estatura (variável maturacional) dos atletas por zonas de posição do campo.

\section{PROCEDIMENTOS METODOLÓGICOS}

Esse estudo foi aprovado pelo COEP da Universidade Federal de Minas Gerais sob o parecer número 0221.0.203.000-11.

\section{Amostra}

A amostra foi composta pelos dados referentes à data de nascimento e estatura de todos os 1493 atletas inscritos nos campeonatos mundiais de futebol sub 17 ocorridos nos anos de 2007 ( $n=507), 2009$ ( $n=484)$ e 2011( $n=502)$. Essa competição envolve atletas com idades entre 16 e 17 anos.

\section{Procedimentos}

Seguindo o método empregado em estudos anteriores ${ }^{16,17}$, utilizou-se como ferramenta de captação dos dados o site oficial da FIFA ${ }^{\circ}$. <www.fifa.com>. Para a comparação das datas de nascimento, os jogadores foram divididos em quatro grupos: Q1 (primeiro quartil de data de nascimento), Q2 (segundo quartil de data de nascimento), Q3 (terceiro quartil de data de nascimento) e Q4 (quarto quartil de data de nascimento), de acordo com o mês de nascimento.

O Quartil de data de nascimento é considerado a divisão do ano em quatro partes, em que, no caso do futebol, independentemente se o atleta está no primeiro ou no segundo ano da categoria, o $1^{\circ}$ quartil representa o grupo de atletas nascidos entre os meses Janeiro; Fevereiro e Março, o $2^{\circ}$ quartil corresponde aos atletas nascidos entre os meses de Abril; Maio e Junho, o $3^{\circ}$ quartil é composto pelos nascidos em Julho, Agosto e Setembro e o $4^{\circ}$ quartil pelos nascidos em Outubro; Novembro e Dezembro ${ }^{6,8}$.

Para a posterior comparação das estaturas dos jogadores, os dados referentes a cada quartil foram subdivididos em quatro grupos, de acordo com os setores do campo em que eles atuavam. Os grupos apresentados foram: goleiros, defensores, meio campistas e atacantes.

\section{Análise Estatística}

A avaliação das diferenças entre os quartis de data de nascimento dos atletas foi realizada através do teste de Qui-Quadrado, com nível de significância de $\mathrm{p}<0,05$. Esse procedimento foi realizado no pacote estatístico SPSS ${ }^{\circ}$ 17.0.

Para a identificação de onde estariam às possíveis diferenças encontradas entre os quartis de data de nascimento dos atletas, foi empregada a 
análise de proporção $2 \times 2$. Nesta análise, foi utilizada a correção de Bonferroni, que altera o nível de significância (p), com a finalidade de evitar erros derivados de múltiplas comparações. O nível de significância corrigido após esse procedimento foi de $\mathrm{p}<0,0083$. Esse procedimento foi realizado no pacote estatístico Minitab"15.0 para Windows.

Para a comparação das médias das estaturas dos atletas, tanto por campeonato avaliado (2007, 2009 e 2011), quanto por zona de posição do campo (goleiros, defensores, meio-campistas e atacantes), foi empregada a análise de variância ANOVA em esquema fatorial $2 \times 2$ e posteriormente, aplicado o post-hoc de Bonferroni, com o valor de p ajustado para $\mathrm{p}<0,0167$. Esses procedimentos foram realizados no pacote estatístico SPSS ${ }^{\circ}$ 17.0.

Foi utilizada, também, a análise de correlação de Spearman para avaliar possíveis relações entre a estatura e o quartil de data de nascimento dos atletas. O nível de significância adotado foi de $\mathrm{p}<0,05$. Esse procedimento foi realizado no pacote estatístico SigmaPlot ${ }^{\circ} 11.0$.

\section{RESULTADOS}

São apresentados na figura 1 a distribuição das datas de nascimento dos atletas, nas tres competições, por quartis de data de nascimento.

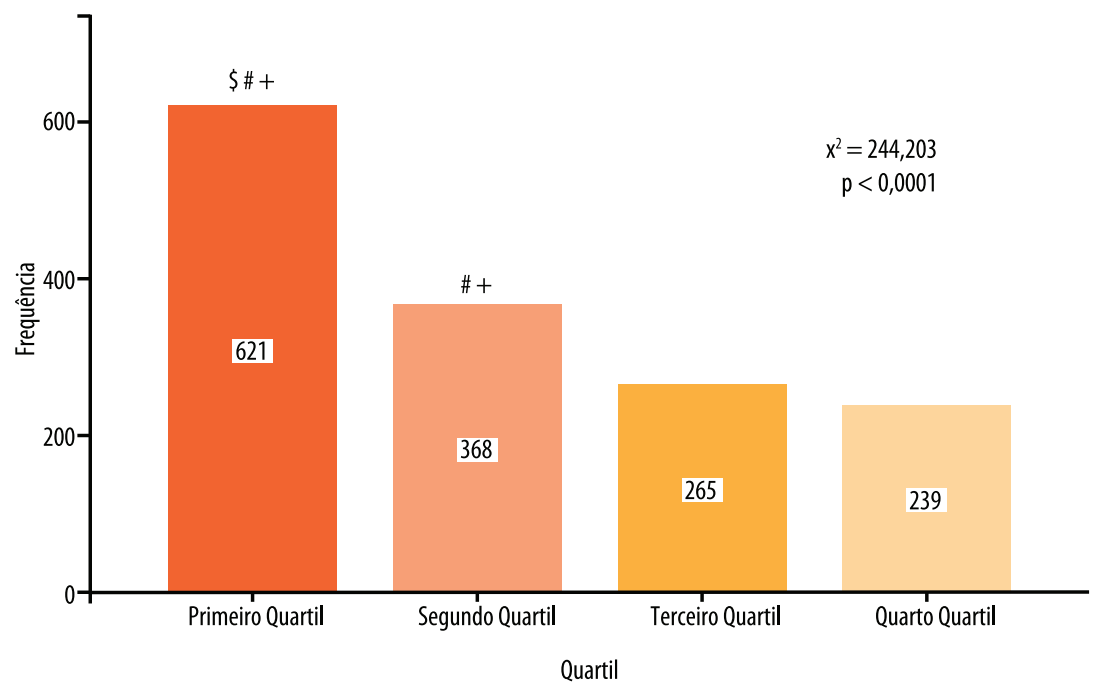

Figura 1. Comparação por meio do teste de qui-quadradado da estratificacão por quartil de data de nascimento dos atletas participantes dos campeonatos mundiais sub 17 em 2007, 2009 e 2011.

$\$$ - diferença significativa para 0 segundo quartil. \#- diferença significativa para 0 terceiro quartil. + - diferença significativa para 0 quarto quartil

Conforme observado, percebe-se que o primeiro quartil de data de nascimento apresenta diferença significativa em relação aos demais quartis de data de nascimento. Observa-se que $41,5 \%$ dos atletas nasceram nesse quartil. O segundo quartil também apresentou diferença significativa em relação ao terceiro e quarto quartis.

Tendo como base estes dois resultados expostos acima, pode-se verificar que nos campeonatos sub-17 destas 3 competições há uma predominância de atletas participantes nascidos no $1^{\circ}$ semestre do ano. 
Em contrapartida, o quarto quartil de data de nascimento é o menos expressivo, agrupando apenas cerca de $16 \%$ dos atletas participantes das competições analisadas. O segundo apresenta $24,5 \%$ dos atletas e o terceiro agrupa $18 \%$.

Os resultados da análise de correlação de Spearman entre estatura e o quartil de data de nascimento por competição não foram significativos para os campeonatos de 2007 ( $r h o=-0,060 \mathrm{p}=0,178)$ e 2009 (rho $=-0,051$ $\mathrm{p}=0$,258). O resultado do campeonato 2011 , apesar de apresentar correlação significativa ( $\mathrm{rho}=-0,122 \mathrm{e} \mathrm{p}=0,006$ ), apresentou uma fraca intensidade de associação entre essas variáveis.

Já a comparação das estaturas dos atletas nascidos nos diferentes quartis da data de nascimento, por campeonato avaliado, os resultados da análise de variância ANOVA em esquema fatorial 2x2 indicam que não existe interação entre as variáveis "campeonato" e "quartil da data de nascimento" ( $\mathrm{F}=0,331$ e $\mathrm{p}=0,921)$. Logo, a comparação das estaturas dos atletas envolvendo as variáveis "Campeonato" e "Quartil da data de nascimento" foram tratadas de maneira isolada.

Com relação a variável “campeonato", não foi identificado efeito principal ( $\mathrm{F}=0,919$ e $\mathrm{p}=0,399)$. Entretanto, observou-se que houve efeito significativo para a variável quartil da data de nascimento $(\mathrm{F}=5,594$ e p=0,001), indicando que a estatura média dos atletas nascidos no segundo quartil foi superior à estatura média dos atletas nascidos no terceiro e quarto quartis de data de nascimento (Tabela 1).

Tabela 1. Comparação das médias da estatura por campeonato e quartil da data de nascimento

\begin{tabular}{lccccccc}
\hline & \multicolumn{2}{c}{2007} & \multicolumn{2}{c}{2009} & \multicolumn{2}{c}{2011} & Média - Quartil \\
\hline Primeiro & $1,77 \pm 0,06$ & $(\mathrm{n}=209)$ & $1,77 \pm 0,07$ & $(\mathrm{n}=212)$ & $1,78 \pm 0,06$ & $(\mathrm{n}=200)$ & $1,77 \pm 0,03$ \\
Segundo & $1,77 \pm 0,06$ & $(\mathrm{n}=117)$ & $1,78 \pm 0,07$ & $(\mathrm{n}=122)$ & $1,78 \pm 0,07$ & $(\mathrm{n}=129)$ & $1,78 \pm 0,04^{\mathrm{a}, \mathrm{b}}$ \\
Terceiro & $1,76 \pm 0,06$ & $(\mathrm{n}=99)$ & $1,76 \pm 0,07$ & $(\mathrm{n}=77)$ & $1,76 \pm 0,06$ & $(\mathrm{n}=89)$ & $1,76 \pm 0,04$ \\
Quarto & $1,76 \pm 0,06$ & $(\mathrm{n}=82)$ & $1,76 \pm 0,07$ & $(\mathrm{n}=73)$ & $1,76 \pm 0,06$ & $(\mathrm{n}=84)$ & $1,76 \pm 0,04$ \\
\hline
\end{tabular}

$a=$ Diferença significativa para o terceiro quartil $(p<0,0167)$

$\mathrm{b}=$ Diferença significativa para 0 quarto quartil $(\mathrm{p}<0,0167)$

A Tabela 2 apresenta a reorganização das médias de estatura por quartil da data de nascimento dos atletas em função da zona de posição do campo.

Tabela 2. Média da estatura dos atletas por zonas de posição do campo.

\begin{tabular}{lllllllll}
\hline & Goleiros & & Defensores & \multicolumn{3}{l}{ Meio-Campistas } & \multicolumn{1}{l}{ Atacantes } \\
\hline Primeiro & $1,83 \pm 0,04 \quad(n=84)$ & $1,78 \pm 0,06$ & $(n=190)$ & $1,75 \pm 0,06$ & $(n=216)$ & $1,76 \pm 0,06$ & $(n=131)$ \\
Segundo & $1,83 \pm 0,06$ & $(n=62)$ & $1,78 \pm 0,06$ & $(n=128)$ & $1,74 \pm 0,06$ & $(n=114)$ & $1,77 \pm 0,05$ & $(n=64)$ \\
Terceiro & $1,83 \pm 0,05$ & $(n=29)$ & $1,76 \pm 0,06$ & $(n=79)$ & $1,74 \pm 0,06$ & $(n=88)$ & $1,75 \pm 0,06$ & $(n=69)$ \\
Quarto & $1,82 \pm 0,05 \quad(n=40)$ & $1,76 \pm 0,07$ & $(n=76)$ & $1,73 \pm 0,05$ & $(n=67)$ & $1,75 \pm 0,06 \quad(n=56)$ \\
\hline
\end{tabular}

Na comparação das estaturas dos atletas por zona de posição do campo, os resultados da análise de variância ANOVA em esquema fatorial 2x2 indicam que não houve interação significativa entre as variáveis "zona de 
posição do campo" e "quartil de nascimento" ( $\mathrm{F}=0,665$ e p= 0,741). Logo, a comparação das estaturas dos atletas envolvendo as variáveis "zona de posição do campo" e "quartil de nascimento" foi realizada de forma isolada.

Com relação à variável "quartil de data de nascimento", foi identificado efeito principal ( $\mathrm{F}=5,237$ e $\mathrm{p}=0,001)$, em que o primeiro e segundo quartis apresentaram estatura média superior ao quarto (tabela 3 ).

Tabela 3. Comparação das médias da estatura por quartil de data de nascimento

\begin{tabular}{lc}
\hline Quartil de data de nascimento & Média \\
\hline Primeiro & $1,78 \pm 0,03^{\mathrm{a}}$ \\
Segundo & $1,78 \pm 0,03^{\mathrm{a}}$ \\
Terceiro & $1,77 \pm 0,04$ \\
Quarto & $1,76 \pm 0,04$ \\
\hline
\end{tabular}

$a=$ Diferenças significativas na estatura a favor dos primeiro e segundo quartis quando comparados com 0 quarto quartil. $(p<0,016)$

Também foi identificado efeito principal para a variável "zona de posição do campo" ( $\mathrm{F}=83,872$ e $\mathrm{p}=0,001)$. Observa-se pela Tabela 4 que os goleiros apresentaram estatura média superior aos defensores, meio-campistas e atacantes. Já os defensores e os atacantes apresentaram uma estatura média superior aos meio-campistas.

Em síntese, estes resultados demonstram uma maior estatura dos goleiros em detrimento as demais posições e uma menor estatura dos atletas que atuam no meio campo em comparação com goleiros, defensores e atacantes.

Tabela 4. Comparação das médias da estatura por zona de posição do campo

\begin{tabular}{lc}
\hline Zona de posição do campo & Média \\
\hline Goleiros & $1,83 \pm 0,05^{\mathrm{a}}$ \\
Defensores & $1,77 \pm 0,03^{\mathrm{b}}$ \\
Meio-Campistas & $1,74 \pm 0,03$ \\
Atacantes & $1,76 \pm 0,04^{\mathrm{c}}$ \\
\hline
\end{tabular}

a = diferença significativa na estatura a favor dos goleiros na comparação com defensores, meio-campistas e atacantes $(p<0,016) ; b=$ diferença significativa na estatura a favor dos defensores na comparação com os meio-campistas $(p<0,016) ; c=$ diferença significativa na estatura a favor dos atacantes na comparação com os meio-campistas $(\mathrm{p}<0,016)$.

\section{DISCUSSÃO}

Esse estudo teve como objetivo analisar a distribuição das datas de nascimento dos atletas de futebol participantes do mundial sub-17, nas edições 2007, 2009 e 2011, e verificar a diferença na estatura (variável maturacional) dos atletas por zonas de posição do campo.

Com isso, observa-se que a detecção e a escolha de prováveis talentos na categoria de base do futebol é tendenciosa para aqueles indivíduos nascidos no início do ano de competição e que apresentem uma possível maturação física avançada ${ }^{14}$. No presente estudo, foi possível observar uma assimetria na distribuição das datas de nascimento dos atletas, que apresentou uma 
alta representação proporcional de atletas nascidos no primeiro quartil da data de nascimento e uma consequente baixa representação proporcional de atletas nascidos no quarto quartil da data de nascimento. Cabe ressaltar que esta amostragem de atletas sub 17 representa a elite do futebol mundial na categoria e retrata um quadro fidedigno de como os efeitos da idade relativa estão presentes nas principais seleções mundiais de futebol e que, muito provalvemente, irão se manifestar também nas competições adultas da modalidade. De acordo com Williams e Reilly ${ }^{18}$ e Reilly et al. ${ }^{19}$, a categoria de base é o espelho do esporte profissional, logo, atletas que servem às categorias de base das seleções nacionais de seus países tem uma maior probabilidade de servirem às seleções adultas nacionais.

Outro fator importante que reforça os resultados encontrados dizem respeito à grande competitividade da modalidade, e também, à qualidade da competição avaliada. Sabe-se que a competitivade e o grau de dificuldade de competição são variáveis que maximizam ou minimizam os efeitos da Idade Relativa ${ }^{11,20}$.

Esses resultados corroboram estudos anteriores, realizados com jovens atletas de futebol de diferentes nacionalidades e contextos ${ }^{5,7}$. O que se observa nestes estudos, anteriormente citados, também foi mapeado nas metanálises realizadas por Musch e Grondin ${ }^{11}$ e Coubley et al. ${ }^{21}$. Analisando os resultados apresentados nestas revisões sobre o efeito da idade relativa no futebol ${ }^{11,21}$, observa-se que na categoria de base, independentemente do tipo e qualidade da competição, existe uma predominância de atletas de $1^{\circ}$ semestre e uma sub representatividade de atletas de quarto quartil.

Dentre as possíveis explicações para esses resultados, pode-se citar a maturação precoce, que pode resultar em vantagens, principalmente, em modalidades esportivas coletivas em que se destaque o tamanho corporal e a estatura ${ }^{22}$. Logo, a análise da variável maturacional estatura dos atletas, demonstra que a maturação representa, de fato, uma importante variável que influencia a seleção de atletas para o futebol ${ }^{23}$. Contudo, como as análises de variância ANOVA em esquema fatorial 2x2 não apresentaram interação entre as variáveis independentes estatura por quartil de nascimento e as variáveis dependentes (ano da competição x zona de posição de campo), o que nos remete a uma discussão dos efeitos maturacionais ligados à estatura por quartil em comparação com as outras variáveis dependentes de forma isolada.

Primeiramente, pode-se observar que quando agrupados por campeonato, os atletas nascidos no segundo quartil de data de nascimento apresentaram estatura média superior aos atletas nascidos no terceiro e quarto quartis de data de nascimento. Esse resultado corrobora, em parte, os resultados encontrados por Carling et al. ${ }^{23}$ que ao avaliarem as características antropométricas de jovens atletas de futebol nascidos nos diferentes quartis do ano competitivo, também encontraram diferenças significativas com relação à altura dos atletas, sendo que essa variável maturacional também foi superior em atletas de segundo quartil de data de nascimento, quando comparados com atletas de quarto quartil de data 
de nascimento. Os autores, acima citados, também econtraram diferenças em atletas de primeiro quartil, quando comparados com atletas de terceiro o quarto quartil o que não foi observado neste estudo.

Com base nos resultados supracitados, e na maior representatividade proporcional apresentada pelo segundo quartil da data de nascimento, quando comparado com o quarto quartil da data de nascimento (Figura 1), pode-se inferir que a maior tendência de seleção de atletas de segundo quartil, em detrimento da seleção de atletas do quarto quartil da data de nascimento, pode, em parte, ser explicada pela maior probabilidade de existir uma diferença de estatura entre esses grupos, já que tanto no presente estudo, quanto no estudo de Carling et al. ${ }^{23}$, atletas das categorias de base do futebol nascidos no segundo quartil da data de nascimento apresentaram estatura média superior ao quarto quartil de data de nascimento.

Os resultados da segunda análise de variância que agrupou os atletas nascidos nos diferentes quartis de data de nascimento, pelas diferentes zonas de posição do campo (Tabela 4), demonstraram que os atletas nascidos mais afastados do início do ano de seleção (quarto quartil de data de nascimento) apresentaram sua estatura média significativamente inferior aos atletas nascidos mais próximos ao início do ano de seleção (primeiro e segundo quartis da data de nascimento). Esses resultados corroboram os achados de Delorme e Raspaud ${ }^{24}$, que encontraram diferenças na estatura de atletas de basquetebol da categoria sub 17, nascidos no primeiro e segundo quartis da data de nascimento, quando comparados com atletas nascidos no quarto quartil da data de nascimento. Até o presente momento, não foi possível identificar na literatura estudos correlacionando o quartil de nascimento, a estatura e a posição em que o atleta atua em campo para que fosse possível uma comparação dentro da própria modalidade. Estudo de Folgado et al. ${ }^{25}$ comparararam somente o quartil de nascimento com a posição em campo e observararam que essa variável influencia a seleção de defensores e meio-campistas.

Logo, os resultados supracitados, juntamente com os resultados da representatividade proporcional (Figura 1), que indicam que atletas nascidos no primeiro e segundo quartis de data de nascimento apresentam representatitivade proporcional superior àquela apresentada pelos atletas de quarto quartil de data de nascimento, apontam que nas modalidades em que o tamanho corporal representa uma importante variável para o sucesso, há uma forte tendência, nas categorias de base, de seleção de atletas nascidos mais próximos ao início do ano de seleção, já que esses atletas tendem a apresentar uma maturação física mais desenvolvida (evidenciada pela diferença na estatura). Essa tendência de seleção já é observada, conforme demonstrado pelo estudo de Nevill et al. ${ }^{26}$, que apontou que atletas de futebol pertencentes a equipes de elite são maiores do que aqueles pertencentes a equipes consideradas não-elite. Entretanto, esta relação não havia sido avaliada em seleções nacionais de futebol na categoria de base, onde pressupõe-se que o rigor nos critérios para a convocação de atletas seja mais elevado em função destes atletas disputarem competições mundiais promovidas pela FIFA. 
Com isso, observa-se que atletas nascidos mais afastados ao início do ano de seleção (quarto quartil da data de nascimento), além de serem preteridos durante o processo de formação (categorias de base), apresentam uma estatura inferior. Logo, para o alcance dos mais elevados níveis competitivos, é hipotetizado por Schorer et al. ${ }^{27}$ que esses atletas desenvolvam um elevado nível de habilidades técnicas, táticas, físicas e psicológicas que os auxilia a se manterem em um processo que lhes é desfavorável. Além disso, também foi demonstrado que esses atletas, quando conseguem atingir os mais elevados níveis competitivos do futebol alemão, ganham maiores salários do que seus pares nascidos anteriormente ${ }^{27}$.

Já a comparação das estaturas das zonas de posições do campo, os resultados do presente estudo corroboram os dados encontrados por Nevil et al. ${ }^{26}$, que também apontam que atletas que atuam na posição de goleiros, defensores e atacantes apresentam uma estatura média superior àquela apresentada pelos meio-campistas. Logo, o quartil da data de nascimento, sendo uma variável que influencia na estatura média dos atletas, pode representar, também, um fator de influência na seleção da posição dos atletas das categorias de base do futebol, principalmente em seus clubes, o que culminará nas seleções nacionais de seus respectivos países. Entretanto, não foram encontrados estudos na literatura que avaliaram a evolução da média da estatura de atletas de futebol pertecentes a seleções nacionais de seus respectivos países.

No que diz respeito à associação entre quartil da data de nascimento e estatura no contexto do futebol de seleções mundiais sub 17, pode-se concluir que em duas edições destas competições (2007 e 2009) de mundiais sub17 não existe nenhum tipo de associação entre as variáveis. Entretanto, no mundial de 2011, esta associação entre as duas variáveis aparece de forma fraca, o que gera a necessidade de uma futura análise no campeonato mundial de seleções de 2013 para verificar se essa tendência de associação entre quartil da data de nascimento e estatura em atletas sub 17 de seleções se confirma ou não e em qual intensidade de associação.

Cabe ressaltar como limitação que este estudo utilizou-se de um banco de dados secundários, disponibilizado pela FIFA, no qual não se consegue precisar com exatidão os protocolos de coleta da estatura destes atletas em suas respectivas seleções. Também podem ter ocorrido erros de digitação das respectivas datas de nascimento e da estatura dos atletas pela FIFA.

Outra limitação que merece destaque diz respeito à avaliação de somente duas variáveis: a data de nascimento e a estatura, em um corte transversal de estudo para avaliar o Efeito da Idade Relativa. Não foi possível, também, verificar o histórico da estatura desses atletas nas categorias subjacentes.

Torna-se importante investigar longitudinalmente, outros parâmetros de rendimento dos atletas de futebol como, por exemplo, as variáveis fisiológicas, técnicas, táticas e psicossociais com a época de nascimento em diferentes faixas etárias, permitindo, assim, um mapeamento mais rigoroso e fidedigno sobre as diferentes variáveis que norteiam o talento esportivo nesta modalidade. 


\section{CONCLUSÃO}

Conclui-se que existem assimetrias na distribuição das datas de nascimento dos jogadores de futebol participantes das edições 2007, 2009 e 2011 do campeonato mundial sub-17. Observa-se que os atletas que participaram destas três competições nasceram, preferencialmente, nos meses de janeiro, fevereiro e março, caracterizando, assim, a preferência dos treinadores de seleções nacionais por atletas nascidos no primeiro quartil da data de nascimento. Nestas três competições avaliadas também é possível identificar uma sub representatividade de atletas de quarto quartil da data de nascimento.

\section{REFERÊNCIAS BIBLIOGRÁFICAS}

1. Musch J, Hay R. The relative age effect in soccer: Cross-cultural evidence for a systematic discrimination against children born late in the competition year. Soc Sport J 1999;16(1):54-64.

2. Simmons C, Paull GC. Season-of-birth bias in association football. J Sports Sci 2001;19:677-86.

3. Glamser FD, Vincent J. The relative age effect ammong elite American youth soccer players. J Sport Behav 2004;27(1):31-8.

4. Vaeyens R, Philippaerts RM, Malina RM. The relative age effect in soccer: A match-related perspective. J Sports Sci 2005;23(7):747-56.

5. Del Campo DGD, Vicedo JCP, Villora SG, Jordan ORC. The relative age effect in youth soccer players from Spain. J Sports Sci Med 2010;9:190-8.

6. Costa VT, Simim M, Noce F, Costa IT, SamulskI DM, Moraes LCCA. Comparison of relative age of elite athletes participating in the 2008 Brazilian soccer championship series A and B. Motricidade 2009;5:35-8.

7. Helsen WF, Winckel JV, Williams AM. The relative age effect in youth soccer across Europe. J Sports Sci 2005;23(6):629-36.

8. Delorme N, Boiché J, Raspaud M. Relative age and dropout in French male soccer. J Sports Sci 2010; 28:717-722.

9. Helsen WF, Hodges NJ, Winckel JV, Starkes JL. The roles of talent, physical precocity and practice in the development of soccer expertise. J Sports Sci 2000;18:727-36.

10. Williams AM. Perceptual skill in soccer: Implications for talent identification and development. J Sports Sci 2000;18:737-50.

11. Musch J, Grondin S. Unequal Competition as an Impediment to Personal Development: A Review of the Relative Age Effect in Sport. Dev Rev 2001;21(2):147-67.

12. Edgar S, O'Donoghue P. Season of birth distribution of elite tennis players. J Sports Sci 2006;23(10):1013-20.

13. Penna EM, Moraes LCCA. Efeito relativo da idade em atletas brasileiros de futsal de alto nível. Motriz 2010;16(3):658-63.

14. Hansen L, Klausen K, Bangsbo J, Muller J. Short longitudinal study of boys playing soccer: Parental height, birth height and length, anthropometry, and pubertal maturation in elite and non-elite players. Pediatr Exerc Sci 1999;11:199-207.

15. Gall F, Carling C, Williams M, Reilly, T. Anthropometric and fitness characteristics of international, professional and amateur male graduate soccer players from an elite youth academy. J Sci Med Sport 2010; 13: 90-95.

16. Côté J, Macdonald DJ, Baker J, Abernethy B. When "where" is more important than "when": Birthplace and birth date effects on the achievement of sporting expertise. J Sports Sci 2006;24(10):1065-73.

17. Macdonald DJ, Cheung M, Cöté J, Abernethy B. Place but not date of birth influences the development and emergence of athletic talent in American football. J App Sport Psychol 2009;21:80-90. 
18. Williams AM, REILLY T. Talent identification and development in soccer. J Sports Sci 2000;18:657-77.

19. Reilly T, Bangsbo J, Franks, A. Anthropometric and physiological predispositions for elite soccer. J Sports Sci 2000;18:669-83.

20. Albuquerque MR, Lage GM, Costa VT, Ferreira, RM, Penna EM, Moraes LCCA, et al. Relative age effect in olympic Taekwondo athletes. Percept Motor Skill 2012;114(2):461-8.

21. Coubley S, Baker, J, Wattie N, McKenna J. Annual Age-grouping and athlete development: A Meta-analytical review of Relative Age Effects in Sport. Sport Med 2009; 39(3):235-56.

22. Silva FM, Fernandes L, Celani FO. Desporto de crianças e jovens - um estudo sobre as idades de iniciação. Rev Port Cien Desp 2001;1(2):45-55.

23. Carling C, Gall F, Reilly T, Williams AM. Do anthropometric and fitness characteristics vary according to birth date distribution in elite academy soccer players? Scand J Med Sci Sports 2009; 19: 3-9.

24. Delorme N, Raspaud, M. The relative age effect in Young French basketball players: a study in the whole population. Scand J Med Sci Sports 2009;19:235-42.

25. Folgado HA, Caixinha PF, Sampaio J, Maçãs V. Efeito da idade cronológica na distribuição dos futebolistas por escalões de formação e pelas diferentes posições específicas. Rev Port Cien Desp 2006;6(3):349-55.

26. Nevill A, Holder R, Watts A. The changing shape of "successful" professional footballers. J Sports Sci 2009;27(5):419-26.

27. Schorer J, Cobley S, Büsch D, Bräutigam H, Baker J. Influences of competition level, gender, player nationality, career stage and playing position on relative age effects. Scand J Med Sci Sports 2009;19:720-30.
Endereço para correspondência

Eduardo Macedo Penna Escola de Educação Física, Fisioterapia e Terapia Ocupacional - UFMG Endereço: Av. Presidente Carlos Luz, 4664.. Bairro Pampulha.

CEP: 31310-250 - Belo Horizonte/ MG. Brasil.

Email:em.penna@yahoo.com.br 